\title{
Impact of the antenna cavity on in-body propagation and channel characteristics between capsule endoscope and on-body antenna
}

\author{
Mariella Särestöniemi \\ Center for Wireless Communications, \\ Faculty of Information Technology and \\ Electrical Engineering \\ University of Oulu \\ Oulu, Finland \\ mariella.sarestoniemi@oulu.fi
}

Markus Berg

Center for Wireless Communications,

Faculty of Information Technology and

Electrical Engineering

University of Oulu

Oulu, Finland

markus.berg@oulu.fi

\author{
Carlos Pomalaza Raez \\ Department of Electrical and Computer \\ Engineering \\ Purdue University \\ Fort Wayne, Indiana 46805, USA \\ cpomalaz@purdue.edu
}

Matti Hämäläinen

Center for Wireless Communications,

Faculty of Information Technology and Electrical Engineering

University of Oulu

Oulu, Finland

matti.hamalainen@oulu.fi

\author{
Chaïmaâ Kissi \\ Electronics and Telecommunication \\ Systems Research Group, National \\ School of Applied Sciences (ENSA) \\ Ibn Tofail University \\ Kenitra, Morocco \\ chaimaakissi1@gmail.com
}

Jari Iinatti

Center for Wireless Communications,

Faculty of Information Technology and Electrical Engineering

University of Oulu

Oulu, Finland

jari.iinatti@oulu.fi

\begin{abstract}
This paper presents a study on the impact of the antenna cavity on the in-body propagation and ultra wideband wireless body area networks (UWB-WBAN) radio channel characteristics between the capsule endoscope and the on-body antenna. The study is conducted with CST Studio Suite simulations and one of its anatomical voxel models. A simplified capsule model and two different on-body antennas designed for low-band UWB in-body communications are used in this study. The first antenna has a smaller cavity while the second antenna has a larger cavity. The radio channel characteristics between a capsule endoscope model and an on-body antenna are evaluated with the cavity and without the cavity in frequency and time domains. Furthermore, 2D power flow representations are studied to get insight how the presence and absence of the cavity changes the signal propagation within the tissues. It is found that with the antenna having a smaller cavity, the cavity impact depends on the on-body antenna location respect to the abdominal muscle layers. Instead, the antenna having a larger cavity enables to capture multipath components from the wider area resulting in stronger signal regardless where the antenna is located.
\end{abstract}

Keywords-antenna cavity, directive antenna, capsule endoscope, ultra wideband, wireless body area networks,

\section{INTRODUCTION}

Implant communications has been under an intensive study in recent years. Especially capsule endoscopy, which is a non-invasive, painless, and reliable method to investigate small intestine thoroughly, has been an actively investigated topic. [1]-[3]

One of the main challenges related to the implant communications is high propagation loss in the tissues [4]. The loss is higher at higher frequencies and thus, the first capsules were designed for $\mathrm{MHz}$ frequencies [2]. However, applying ultra wideband (UWB) technology for capsule endoscopy has been found to provide several advantages, such as high-resolution images, low power, and simple transceivers [1]. Thus, the challenges relating to the high propagation loss should be solved. One method to overcome problems related to high propagation losses is to use highly directive on-body antennas which focus the radiation towards the body and hence may strengthen the communication link between the implant and the on-body device. The use of the antenna cavities is one commonly used approach to enhance directivity of the on-body antenna towards the body. A properly designed cavity may enhance antenna's directivity towards the body significantly [5]-[13].

Back to literature, up to date, all published works investigating wireless capsule endoscopy communication systems at 3.5-4.5 GHz frequency band have used large external antennas [5]-[8]. Besides, they have lower measured gain than the cavity-backed antenna object of this work. Additionally, further information regarding their radiation pattern and directivity is lagging in all published papers. The on-body external antennas used in this paper were properly designed for in-body communications by providing a normal directivity to the body surface. In fact, the cavity size seems to restrict the practical use of wearable antennas for clinical scenarios. Nonetheless, few approaches can be followed to reduce significantly the cavity size, among them we cite: to fill the current air-filled cavity by resin material, which is known by its high dielectric properties, hence the size can be compact and more suitable to wearable use. On the other hand, the cavity features can be saved by replacing the backed cavity by a small-sized reflector, as can be seen in [12]. Therefore, the present antennas in this investigation are suitable for this study to present preliminary and initial results for coming studies. The overall cavity dimensions for both antennas have a great impact on the resonant frequency, covered bandwidth, gain and the radiation pattern shape. Detailed analysis of how the cavity dimensions of both antennas affect these output parameters are detailed and can be found in [9], [11], [12].

Impact of the antenna's cavity is studied for antenna characteristics e.g. in [7]-[13]. However, up to author's knowledge, most of the cavity studies focus on the cavity 
impact on the antenna characteristics. There is a lack of studies presenting the impact of the cavity on signal propagation within the tissues and on the radio channel characteristics for the practical scenarios.

This paper presents a simulation -based study on the impact of the antenna cavity on the in-body signal propagation and radio channel characteristics in the context of capsule endoscopy. Two different antennas are used in this study: Antenna 1, originally introduced in [9] has a smaller cavity and Antenna 2, originally introduced in [13] has a larger cavity. CST Studio Suite software [14] and its anatomical voxel model Laura is used in the simulations. A simplified model of the capsule endoscope is set in the voxel model's small intestine and channel simulations are conducted with two different antenna location options. The radio channel characteristics between a capsule endoscope model and an onbody antennas are evaluated with the cavity and without the cavity in frequency and time domains. Furthermore, 2D power flow representations are studied to get insight how the presence and absence of the cavity changes the signal propagation within the tissues. Finally, the impact of the cavity size is also discussed.

This paper is organized as follows: Section II presents the study case describing the simulation model, on-body antennas, and the capsule endoscope model. Section III presents the in-body propagation studies with Antenna 1 with 2D power flow evaluations. Besides, the channel characteristics between the capsule model and the on-body antenna are evaluated in two different antenna location options. Section IV presents similar evaluations with Antenna 2. Summary and Conclusions are given in Section V.

\section{STUDY CASE}

\section{A. Simulation model}

The simulations were conducted using finite integration technique (FIT) -based 3D electromagnetic simulation tool CST Studio Suite [14]. The basic idea of FIT is to calculate the radio propagation by solving the Maxwell's equations in their integral form [15].

Simulations were carried out using a female voxel model Laura, which is presented in Fig. 1a. The resolution of Laura voxel is $1.88 \mathrm{~mm} \times 1.88 \mathrm{~mm} \times 1.88 \mathrm{~mm}$. The simulations were conducted in the small intestine area of the voxel model at the cross-cut level presented in Fig. 1a. The cross-cut of the voxel model is presented in Fig. $1 \mathrm{~b}$.

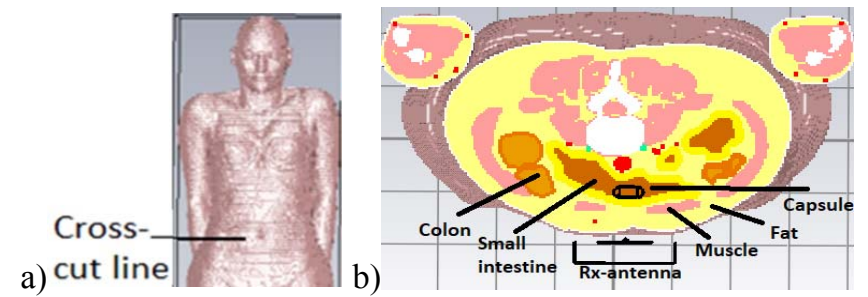

Fig. 1. a) Laura voxel model, b) cross-cut of Laura voxel model.

\section{B. On-body antennas}

Two different on-body antennas designed for implant communication systems are used in this study. Antenna 1, shown in Fig 2a, has a radiator having a form of a ring, and thus it is referred to as a Ring antenna, was originally introduced in [9]. The antenna has a cavity having the size of $\mathrm{x}=83 \mathrm{~mm}, \mathrm{y}=49.5 \mathrm{~mm}$, and $\mathrm{z}=19.62 \mathrm{~mm}$, where $\mathrm{z}$ is towards the body. The size of the antenna itself is $x=47.5 \mathrm{~mm}, y=47.5$ $\mathrm{mm}$. The antenna is designed to work at frequency band 3.754.25 GHz, which meets the IEEE 802.15.6 standards requirements [16]. A detailed description of the antenna, its properties with and without the cavity can be found in [9] and its further extension in [10]. Initial radio channel evaluation studies for capsule endoscope communications can be found in [17], which were further studied more in details in [18], [19].

Antenna 2, shown in Fig. 2b, is also designed for implant communications and was originally presented in [13]. This antenna has a larger cavity size: $x=96 \mathrm{~mm}, y=94 \mathrm{~mm}, z=55$ $\mathrm{mm}$. The size of the antenna is $x=36 \mathrm{~mm}, y=36 \mathrm{~mm}$. One should note that the other side of the cavity has been eliminated. The cavity of such a large size enhances directivity but may restrict the practical solutions. Detailed structure and characteristics of Antenna 2 with and without the cavity are presented in [13].

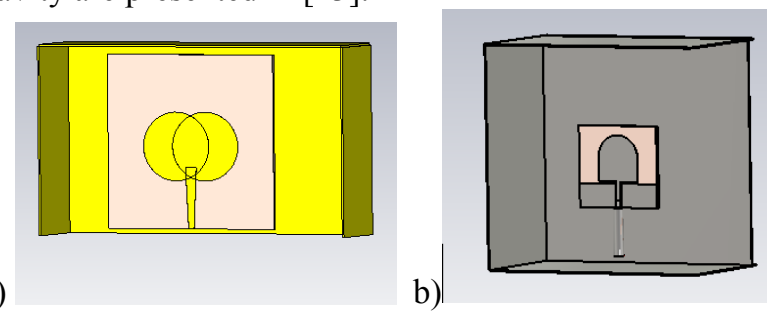

a)

b)

Fig. 2. a) On-body Antenna 1, b) On-body Antenna 2.

\section{Capsule model}

In this study, we use same simplified capsule model as e.g. in [17]- [19], where the details of the model can be found. This section only briefly summarizes the main characteristics of the model.

In the capsule model, an omnidirectional dipole antenna is embedded in plastic capsule shell, which has realistic dimensions: $11 \mathrm{~mm} \times 25 \mathrm{~mm}$, corresponding to the size of the commercial capsules nowadays [1]. The scheme of the dipole antenna and the capsule shell are presented in Fig. 3a-b, respectively. The dipole antenna is designed to work at the frequency of $4 \mathrm{GHz}$ inside the small intestine.

a)

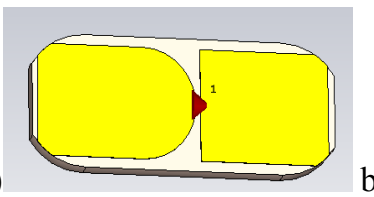

b)

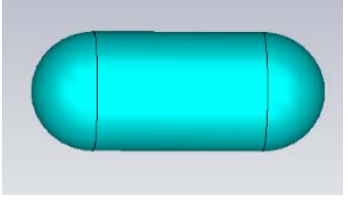

Fig. 3. a) Dipole antenna inside the capsule, b) and the capsule shell.

\section{On-body antenna and capsule endoscope locations}

In this study, two on-body antenna locations are considered: On-body antenna location 1 is on the navel as presented in cross-section Fig. 4a and on-body antenna location 2, presented in Fig. 4b, the antenna is shifted $4 \mathrm{~cm}$ on the right side from the central line. These antenna locations are selected since the propagation between the capsule and on-body antenna is different in these two cases due to different location respect to the muscle layer. More details of the advantages and disadvantages of these on-body 
antenna locations van be studied e.g. in [17]. Antenna-body distance is $4 \mathrm{~mm}$, which correspond to the thin cloth thickness.

The location of the capsule is selected so that the distance between the capsule and on-body antenna is somewhat similar in both on-body antenna location cases. The on-body antenna and the capsule locations are presented in Fig. 4 a-b.

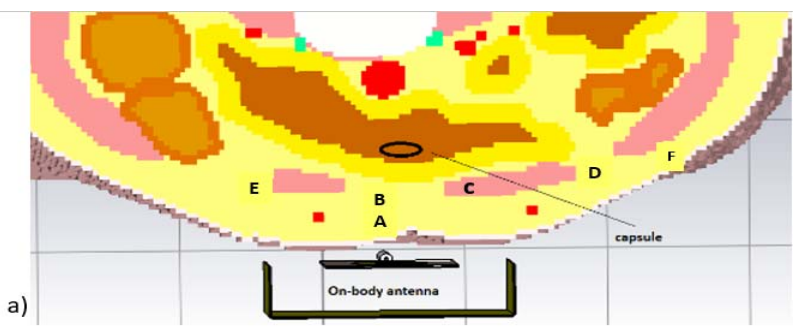

b)

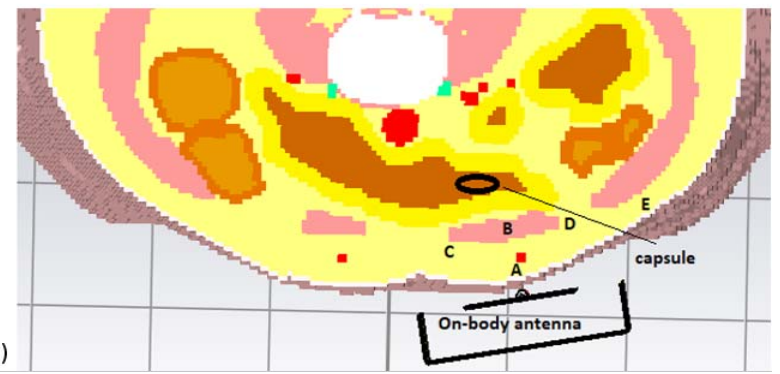

Fig. 4. a) On-body antenna locations 1 and capsule location "middle", b) On-body antenna location 2, capsule location "left".

\section{CHANNEL EVALUATIONS WITH ON-BODY ANTENNA 1}

\section{A. Antenna location 1}

In this section, the impact of the cavity is evaluated in the antenna location 1 and capsule location "middle" with Antenna 1 (Ring antenna). Firstly, we compare the 2D power flows obtained using the Ring antenna with cavity and Ring antenna without the cavity at $4 \mathrm{GHz}$ in Figs. $5 \mathrm{a}-\mathrm{b}$, respectively. The plotted power flow range is $0--70 \mathrm{~dB}$, having $0 \mathrm{~dB}$ on the antenna. Power flow for the Ring antenna with the cavity was presented in [18], but it is repeated here to ease the comparison between the cases with and without the cavity. As it is noted, from Fig. 5a, the cavity clearly directs the power flow towards the body, and also towards the capsule location "middle". Ring antenna without the cavity has more power flow also on the sides and back side, as expected from the radiation pattern presented in [10].

Next the power flow values are evaluated using Ring antenna with and without the cavity at $4 \mathrm{GHz}$ in the points $\mathrm{A}$, B, C, D, E, and F depicted in Fig. 4a. The power flow values are listed in Table I. As expected from the power flow presentations, the power flow values with the cavity are higher in the points $\mathrm{A}, \mathrm{B}$, and $\mathrm{C}$ than without the cavity. However, the difference is maximum $3 \mathrm{~dB}$. Instead, the power values are lower when cavity is used in the points $\mathrm{D}$, $\mathrm{E}$, and $\mathrm{F}$, which are more on the side regions respect to the on-body antenna. The differences are 2-3 dB.

The differences in the power flows can be seen in frequency and time domain channel characteristics presented in Figs. 6a-b, respectively. Fig. 6a presents channel frequency response S21 and the Fig $6 \mathrm{~b}$ presents the channel impulse response which is obtained using inverse fast Fourier transform (IFFT) on the S21 for the whole simulated bandwidth 0-5 GHz. As it can be seen in Fig. 6a, S21 value is approximately $5 \mathrm{~dB}$ higher when cavity is used within the frequency range of interest 3.75-4.25 GHz. In time domain results, presented in Fig. $6 \mathrm{~b}$, the main propagation path (i.e., the impulse response's main peak) obtained with and without the cavity are at the same level, but the main peak is $0.25 \mathrm{~ns}$ wider as the cavity is used. Side peaks are stronger as the cavity is used. For instance, the second peak, which is at the level relevant for the signal detection, is $8 \mathrm{~dB}$ stronger as the cavity is used. Enhanced power flow towards the body strengthen also alternative propagations paths, not only the main path and thus, the side peaks are at higher level in this case. Enhanced power flow may also bring up new propagation paths depending on how different the antenna patterns are between the cases with and without the cavity. For instance, fat tissue is known to be a good propagation channel in terms of lower propagation loss and propagation delay [17], [20]. Thus, strong power flow towards the areas, where outer fat layer is easily accessed from the inner fat layer, may provide strong additional propagation paths for the transmitted signal.

Reflection coefficients for Ring antenna with and without the cavity are presented in Fig. 6c. Interestingly, the antenna matching is better without the cavity. This was also found in [10], where the impact of the cavity on the antenna characteristics is studied more in details. In [10] it was found that the impact of the cavity on the antenna characteristics depends on the location of the on-body antenna: the impact was different on the backside compared to the abdomen side. Thus, we evaluate in the next section the impact of the cavity on the capsule-on-body link at the on-body antenna location 2 and capsule location "front right".
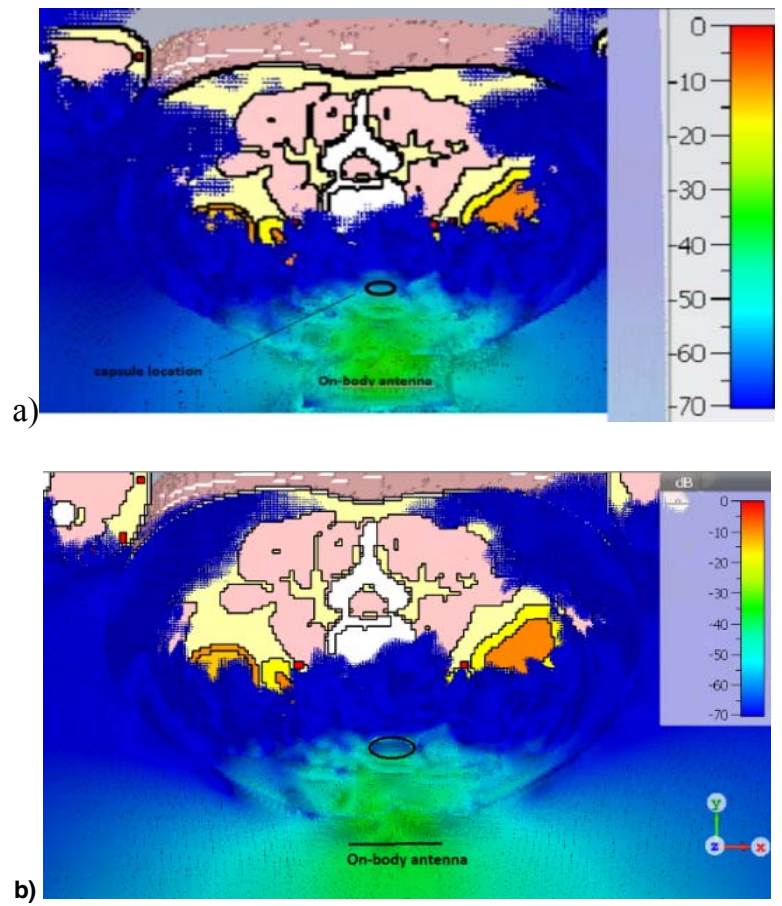

Fig. 5. Power flow at $4 \mathrm{GHz}$ from Ring antenna a) with the cavity and b) without the cavity at the on-body antenna location 1 . 
TABLE I. POWER FLOW VALUES FOR ANTENNA LOCATION 2

\begin{tabular}{|l|l|l|l|l|l|l|}
\hline Location & $\begin{array}{l}\mathbf{A} \\
{[\mathbf{d B}]}\end{array}$ & $\begin{array}{l}\mathbf{B} \\
{[\mathbf{d B}]}\end{array}$ & $\mathbf{C}[\mathbf{d B}]$ & $\mathbf{D}[\mathbf{d B}]$ & $\begin{array}{l}\mathbf{E} \\
{[\mathbf{d B}]}\end{array}$ & $\begin{array}{l}\mathbf{F} \\
{[\mathbf{d B}]}\end{array}$ \\
\hline $\begin{array}{c}\text { With } \\
\text { cavity }\end{array}$ & -34 & -37 & -42 & -57 & -53 & -66 \\
\hline $\begin{array}{c}\text { Without } \\
\text { the cavity }\end{array}$ & -37 & -40 & -43 & -55 & -50 & -64 \\
\hline
\end{tabular}
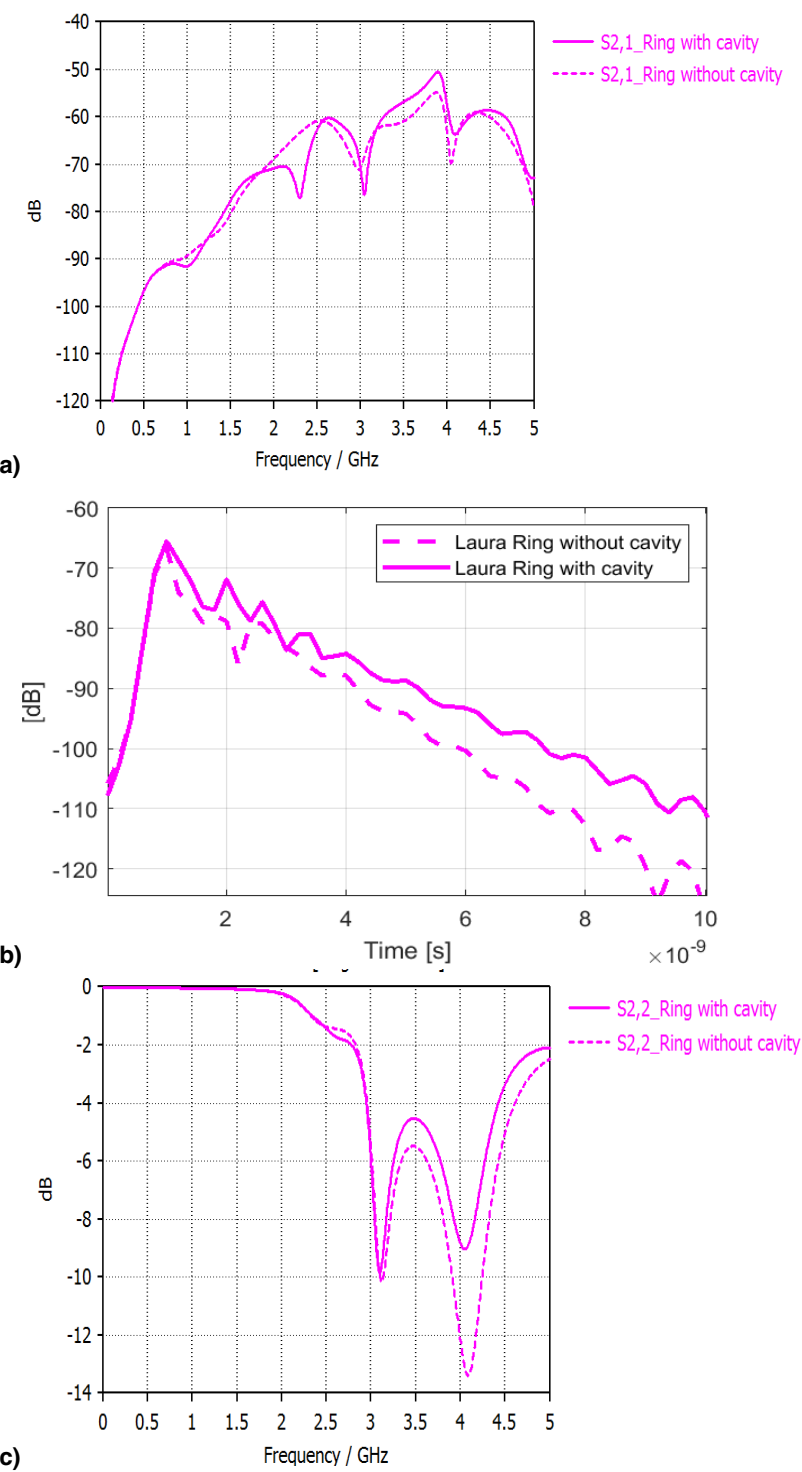

Fig. 6. a) frequency and b) time domain channel characteristics as well as c) antenna reflection coefficients at on-body antenna location 1 using Ring antenna with and without the cavity.

\section{B. Antenna location 2}

As in the case of antenna location 1, we firstly compare the power flows obtained using Ring with and without the cavity as presented in Figs. 7a-b, respectively. Evidently also in this case, power flow towards the body with the cavity clearly stronger in the presence of the cavity. Without the cavity, power flow is spread more on the sides and through the outer fat layer it propagates in the larger area. The area, in which the capsule is located, is surrounded by strong power flows since there is a clear beam towards the area where outer fat joins to the visceral fat layer (between the abdominal muscles). Instead in the case with the cavity, power flow is slightly weaker at the regions of where outer fat joins the visceral fat. This tendency can be found also from the power flow values evaluated in the points $\mathrm{A}, \mathrm{B}, \mathrm{C}, \mathrm{D}$, and $\mathrm{E}$, and which are presented in Table II. Power flow values obtained with the cavity are 5-6 dB larger in the points A and B, which are in the direct line from the antenna towards the SI area. Instead, power flow values in the points which are on the side area, are larger without the cavity. Especially at the point E, the difference is largest: without the cavity, the power flow is $12 \mathrm{~dB}$ larger without the cavity than with the cavity.

TABLE II. POWER FLOW VALUES FOR ANTENNA LOCATION 2

\begin{tabular}{|l|l|l|l|l|l|}
\hline Location & $\mathbf{A}[\mathbf{d B}]$ & $\mathbf{B}[\mathbf{d B}]$ & $\mathbf{C}[\mathbf{d B}]$ & $\mathbf{D}[\mathbf{d B}]$ & $\mathbf{E}[\mathbf{d B}]$ \\
\hline With cavity & -36 & -43 & -53 & -50 & -69 \\
\hline $\begin{array}{l}\text { Without the } \\
\text { cavity }\end{array}$ & -42 & -48 & -49 & -47 & -57 \\
\hline
\end{tabular}

Frequency and time domain channel characteristics are presented in Figs. 8a-b, respectively. The antenna reflection coefficients are included in Fig. 8b a as well. In this case, the impact of the cavity is remarkable smaller, there is only minor differences in the reflection coefficients and path losses in the frequency range of interest. Interestingly, path loss is slightly minor, approximately $2 \mathrm{~dB}$, without the cavity at $3.75-4$ $\mathrm{GHz}$. In time domain, the first two peaks of the impulse responses are at the same level with and without the cavity. At the time range $1.6-2 \mathrm{~ns}$, the impulse response obtained without the cavity is at up to $5 \mathrm{~dB}$ higher level than that obtained without the cavity. The reason behind the slightly stronger channel in the case without the cavity is assumed to be due to the stronger power flow via fat layer.
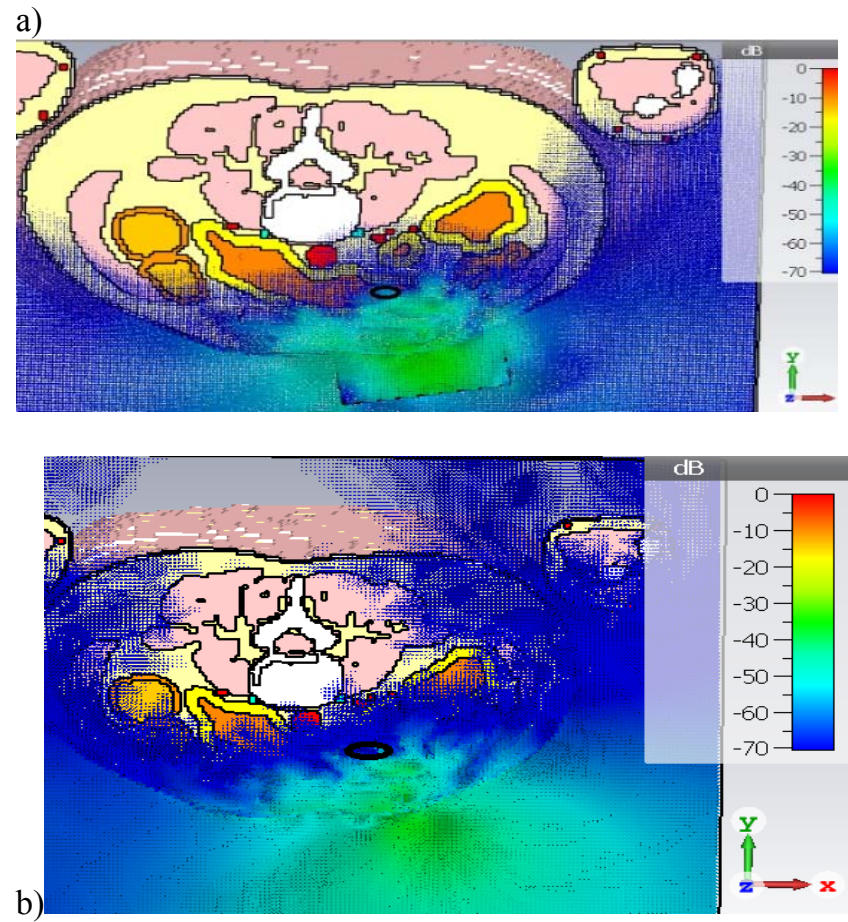

Fig. 7. Power flow at $4 \mathrm{GHz}$ from Ring antenna a) with the cavity and b) without the cavity at the on-body antenna location 2 . 
a)

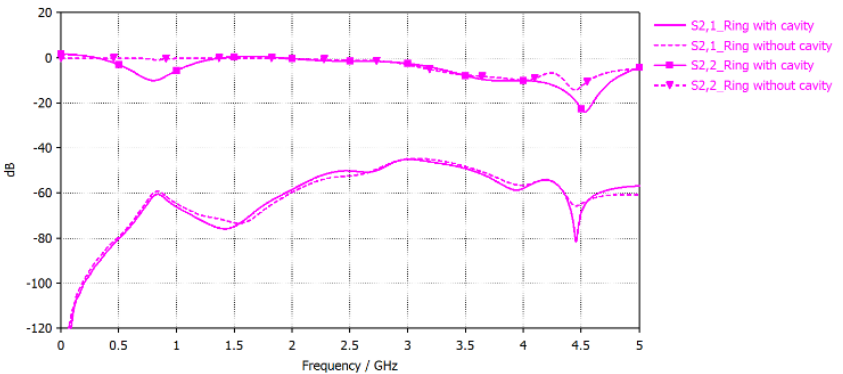

b)

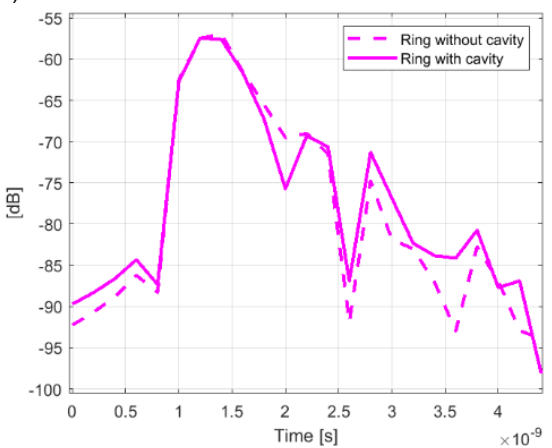

Fig. 8. a) Reflection coeffcients $\mathrm{S} 22 \mathrm{~s}$ and channel responses $\mathrm{S} 21 \mathrm{~s}$, b) time domain channel characteristics for Ring antenna with and without the cavity at antenna location 1 .

\section{CHANNEL EVALUATIONS WITH ON-BODY ANTENNA 2}

In this section, the in-body propagation and channel characteristics between the capsule and the on-body antenna 2 are evaluated in the cases with the cavity and without the cavity. Due to lack of space, only antenna location option 2 is considered in this case.

First, the power flows are studied in Fig. 9a-b for the antenna with and without the cavity, respectively. Also, in this case, the impact of the cavity is clearly visible: power flow is stronger towards the body. In general, almost all the intestine area is covered within the $\mathrm{dB}$ range of interest. The absence of the left cavity edge is noted clearly in Fig. 9a: power flow is strong outside the absent cavity edge. In the case without the cavity, one can note similar stronger power flow on the sides as in the case of Ring antenna.

Next, the frequency and time domain channel characteristics are studied in Fig.10a-b, respectively. Reflection coefficients are included in Fig. 10a. In this case, antenna matching is weak in both cases with the selected antenna-body distance. Within the frequency range of interest, Antenna 2 with the cavity has 2-6 dB smaller path loss than Antenna 2 without the cavity. In time domain, the difference is even larger: at the time instant $2 \mathrm{~ns}$, which corresponds to the second multipath component, the peak level is even $25 \mathrm{~dB}$ larger with the cavity than without the cavity. The difference is due to the fact that the cavity enhances the propagation towards the body. Besides, since the cavity is large, it enables capsule's signal reception from the larger area.

Finally, we compare the impulse responses obtained using Antenna 1 and Antenna 2 with the cavities (presented in Fig. 8b and Fig. 10b) in the antenna location 2 to get an idea about the impact of the cavity size. We can see that the levels of the impulse responses' main peaks are somewhat similar: for antenna 1 the level is $-58 \mathrm{~dB}$ and for antenna 2 the level is $-56 \mathrm{~dB}$. Instead, there is a large difference in the levels of the second paths: $-70 \mathrm{~dB}$ and $-60 \mathrm{~dB}$ for Antenna 1 and Antenna 2, respectively. Evidently, the dimensions of the cavity, its type, and directional characteristics of the antenna have a clearest impact on the number and level of the multipath components that could be captured. In the case of Antenna 1, the second peak of the impulse response is attenuated strongly: it is $-25 \mathrm{~dB}$ below the main peak whereas with the Antenna 2, the difference between the main and second peak levels is only $4 \mathrm{~dB}$.

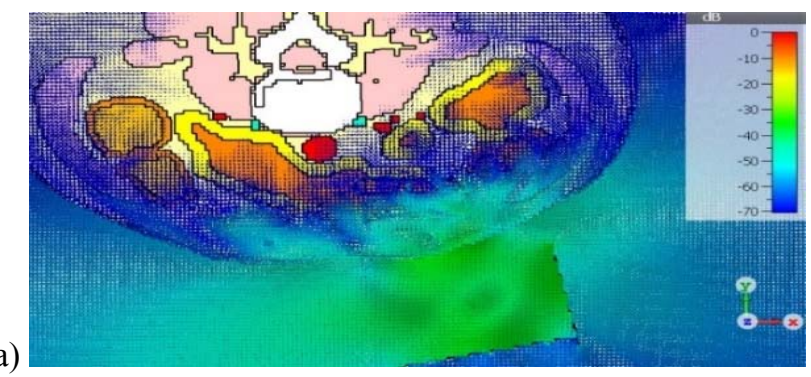

b)

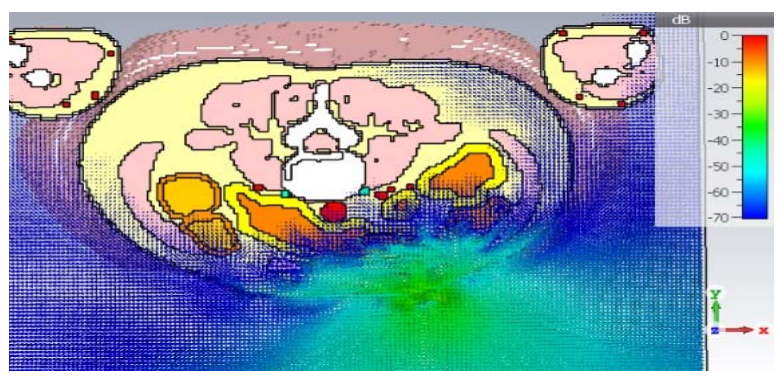

Fig. 9. Power flow at $4 \mathrm{GHz}$ from Antenna 2 a) with the cavity and b) without the cavity at the on-body antenna location 2 .
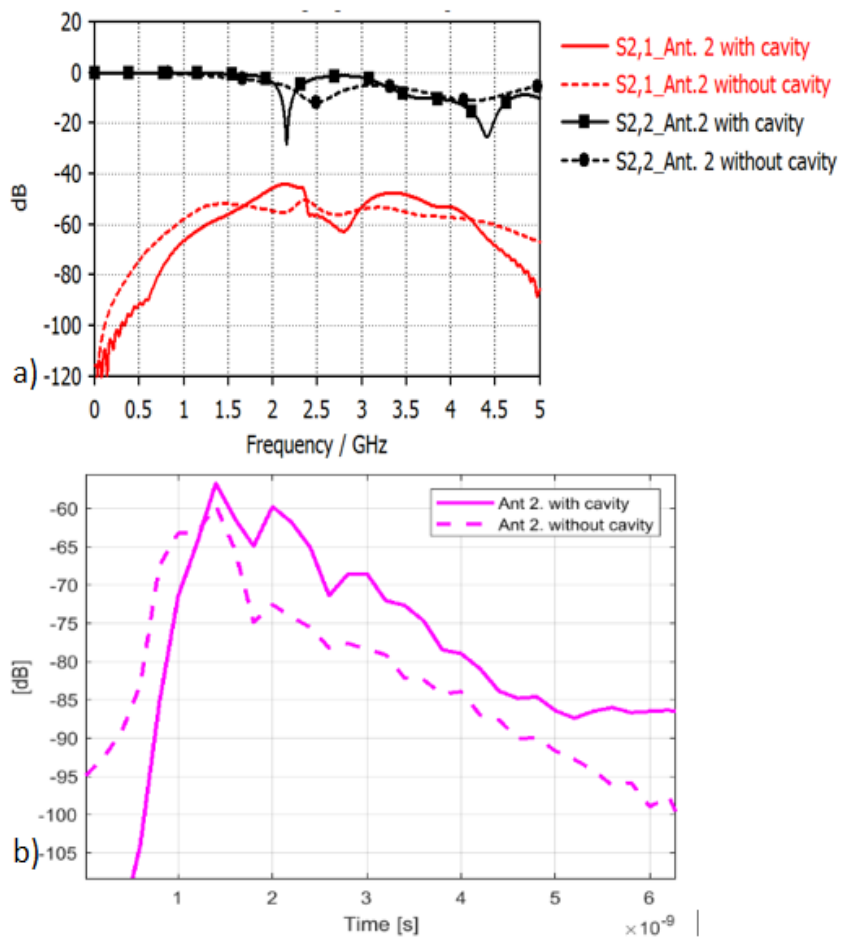

Fig. 10. a) Reflection coeffcients S22s and channel responses S21s, b) time domain channel characteristics for Antenna 2 with and without the cavity. 


\section{SUMMARY AND CONCLUSIONS}

In this paper, the impact of the on-body antenna's cavity for inbody propagation and WBAN channel characteristics was studied using two different on-body antennas designed for inbody communications. These directive antennas had different sizes of the cavities: Antenna 1 had a smaller cavity, Antenna 2 a larger cavity. The radio channel characteristics between a capsule endoscope model and an on-body antenna were evaluated with the cavity and without the cavity in frequency and time domains. Furthermore, 2D power flow representations were studied to get insight how the presence and absence of the cavity changes the signal propagation within the tissues.

It was found that as the antenna cavity is smaller, the impact of the cavity depends on the on-body antenna location respect to the abdominal muscle layers. As the antenna was located on the navel, which is considered the best location for the implant communications, the cavity enhanced the directivity towards the body and thus the channel between the capsule and the on-body antenna improves significantly on the side peaks of the impulse response: $2-20 \mathrm{~dB}$ on the side peaks. However, as the on-body antenna is located above the muscle layer, the power flow towards the sides had more advantage enabling stronger multipath components through the fat layers. Thus, the side peak at the time instant of $2 \mathrm{~ns}$ is at higher level without the cavity. If the cavity size is larger, as in the case of Antenna 2, the cavity enables to capture several multipath components resulting clearly stronger received power level even in the antenna location 2. Hence, in general it can be said that the larger the cavity, the better diversity from the multipath components can be obtained. Nevertheless, larger cavities may be a problem in the practical solutions with werable on-body devices.

As a future work, we aim to study impact of the cavity with several different on-body antennas and on-body antenna locations using voxel models having different size and body constitutions. Besides, the impact of the antenna-body distance on the cavity impact will be studied as well. The studied and proposed antenna is a first prototype of our fruitful investigations. Future studies aiming to diminish its size will be highly regarded in coming papers. One of the solutions to reduce the cavity size is to fill the air-cavity with resin material (of higher dielectric properties) etc. Still further free-space and on-body simulation and measurement investigations will be presented in a coming paper.

\section{ACKNOWLEDGMENT}

This research has been financially supported by the project This work is supported in part by the projects WBAN Communications in the Congested Environments (MeCCE), the Academy of Finland 6Genesis Flagship (grant 318927) and the European Union's Horizon 2020 programme under the Marie Skłodowska-Curie grant agreement No. 872752. Dr. Marko Sonkki is acknowledged for his participation on the on-body antenna design.

\section{REFERENCES}

[1] G. Ciuti; A. Menciassi; P. Dario, “ Capsule Endoscopy: From Current Achievements to Open Challenges", IEEE Reviews in Biomedical Engineering, vol.4, pp. 59-72, 2011.

[2] D. R. Cave, S. Hakimian, and K. Patel, "Current controversies concerning capsule endoscopy," Digestive Diseases and Sciences, vol. 64 , no. 11 , pp. 3040-3047, 2019.

[3] H. Neumann, L. C. Fry, A. Na"gela, and M. F. Neurath ; "Wireless capsule endoscopy of the small intestine: a review with future directions"; Current Opinion in Gastroenterology; Vol 30; Issue 5; pp 463-471, 2014

[4] https://www.itis.ethz.ch/virtual-population/tissue-properties/database

[5] J-. Y. Li; R. Xu; X. Zhang; S-. G. Zhou; and G-. W. Yang, "A Wideband High-Gain Cavity-Backed Low-Profile Dipole Antenna", IEEE Transactions on Antennas and Propagation, vol. 64, pp. 5465 5469, 2016

[6] A. Munir; R. B. V. B. Simorangkir; and F. Kurniawan, "Late-time Ringing Characterization of Cavity-Backed UWB Printed Monopole Antenna", IEEE Conference on Antenna Measurements \& Applications (CAMA), pp. 419 - 422, 2017.

[7] R. Ferreira; J. Joubert; and J. W. Odendaal, "A compact dual-circularly polarized cavity-backed ring-slot antenna", IEEE Transactions on Antennas and Propagation, vol. 65, pp. 364 - 368, 2017.

[8] Q. V. d. Brande; S. Lemey; J. Vanfleteren; and Hendrik Rogier, "Highly-Efficient Impulse-Radio Ultra-Wideband Cavity-Backed Slot Antenna in Stacked Air-Filled Substrate-Integrated-Waveguide Technology", IEEE Transactions on Antennas and Propagation, vol. 66 , pp. $2199-2209,2018$

[9] C. Kissi ; M. Särestöniemi ; T. Kumpuniemi ; M. Sonkki ; S. Myllymäki ; M. N. Srifi and C. Pomalaza-Raez , " Low-UWB Receiving antenna for WCE Localization", ISMICT 2019.

[10] C. Kissi ; M. Särestöniemi ; T. Kumpuniemi ; J-P. Mäkelä, M. Sonkki ; S. Myllymäki ; M. N. Srifi, H. Jantunen, and C. P-. Raez,'"Receiving UWB Antenna for Capsule Endoscope Communications," accepted to be published in PIERS journal in March 2020.

[11] C. Kissi, M. Särestöniemi, S. Myllymäki, M. Sonkki, N. Srifi, H. Jantunen, C. Pomalaza " Dual Band CPW-Fed Double Monopole Antenna for 2.4/5.8 GHz ISM band Medical Applications, " ISAECT 2019.

[12] C. Kissi, M. Särestöniemi, T. Kumpuniemi, S. Myllymäki, M. Sonkki, N. Srifi, H. Jantunen, C. Pomalaza-Raez, "Reflector-Backed Antenna for UWB Medical Applications with On-Body Investigations. International Journal of Antennas and Propagation, 2019, 1-17.

[13] C. Kissi, M. Särestöniemi, T. Kumpuniemi, S. Myllymäki, M. Sonkki, N. Srifi, H. Jantunen, C. Pomalaza-Raez, "High-Directivity Antenna for LOW-UWB BodyArea Networks Applications," ISAECT2018.

[14] CST Studio Suite, [Online]. Available: http://www.cst.com

[15] S. J. Orfanidis, "Electromagnetic Waves and Antennas," http://www.ece.rutgers.edu/ orfanidi/ewa/.

[16] IEEE Standard for Local and metropolitan area networks Part 15.6: Wireless Body Area Networks, pp. IEEE Std 802.15.6-2012, pp. 1 271, 2012.

[17] M. Särestöniemi, C. Pomalaza-Raez, M. Berg, C. Kissi, M. Hämäläinen. J. Iinatti, "In-Body Power Distribution for Abdominal Monitoring and Implant Communications Systems," ISWCS, September 2019

[18] M. Särestöniemi, C. Pomalaza-Raez, M. Berg, C. Kissi, M. Hämäläinen. J. Iinatti, "UWB-WBAN Radio Channel Characteristics between the Endoscope Capsule and On-body Antenna," BodyNets2019, October 2019.

[19] M. Särestöniemi, C. Pomalaza-Raez, M. Berg, C. Kissi, M. Hämäläinen. J. Iinatti, "WBAN channel characteristics between capsule endoscope and receiving directive UWB on-body antennas", IEEE Access, March 2020.

[20] N. B. Asan, E. Hassan, J. Shah, D. Noreland, T. Blokhuis, R. Augustine, "Characterization of the fat channel for intra-body communication at R-band frequencies," Sensors, vol. 18, no. 9, p. 2752,2018 . 